\title{
Comparison of Chemical and Biological Properties of Metal Nanoparticles (Au, Ag) with Metal Oxide Nanoparticles (ZnO- NPs) and their Applications
}

\author{
Wanisa Abdussalam-Mohammed* \\ Department of Chemistry, College of Science, Sebha University, Sebha, Libya
}

\section{A R T I C L E I N F O}

Received: 14 June 2019

Revised: 19 July 2019

Accepted: 23 August 2019

Available online: 25 August 2019

\section{K E Y W O R D S}

Gold nanoparticles Silver nanoparticles Zinc oxide nanoparticles

Toxicity growth

Antibacterial activity

Stability

Capping agents

\section{A B S T R A C T}

Biological application of nanoparticles (NPs) is a rapidly developing area of nanotechnology, providing new possibilities in the diagnosis and treatment of human diseases. Nowadays, NPs have shown abilities to be used as alternative treatment for difficult diseases. Therefore, it is important to understand NP chemistry, preparation, interaction and possible mechanisms involved in its interaction with cancer cells and bacterial membranes. This study reports comparison between $\mathrm{Au}, \mathrm{Ag}$ and $\mathrm{ZnO}$ NPs, and discusses their toxicity and physicochemical properties. In addition, this work aims to review different strategies of surface modification and functionalization of colloidal nanoparticles.

\section{G R A P H I C A L A B S T R A C T}

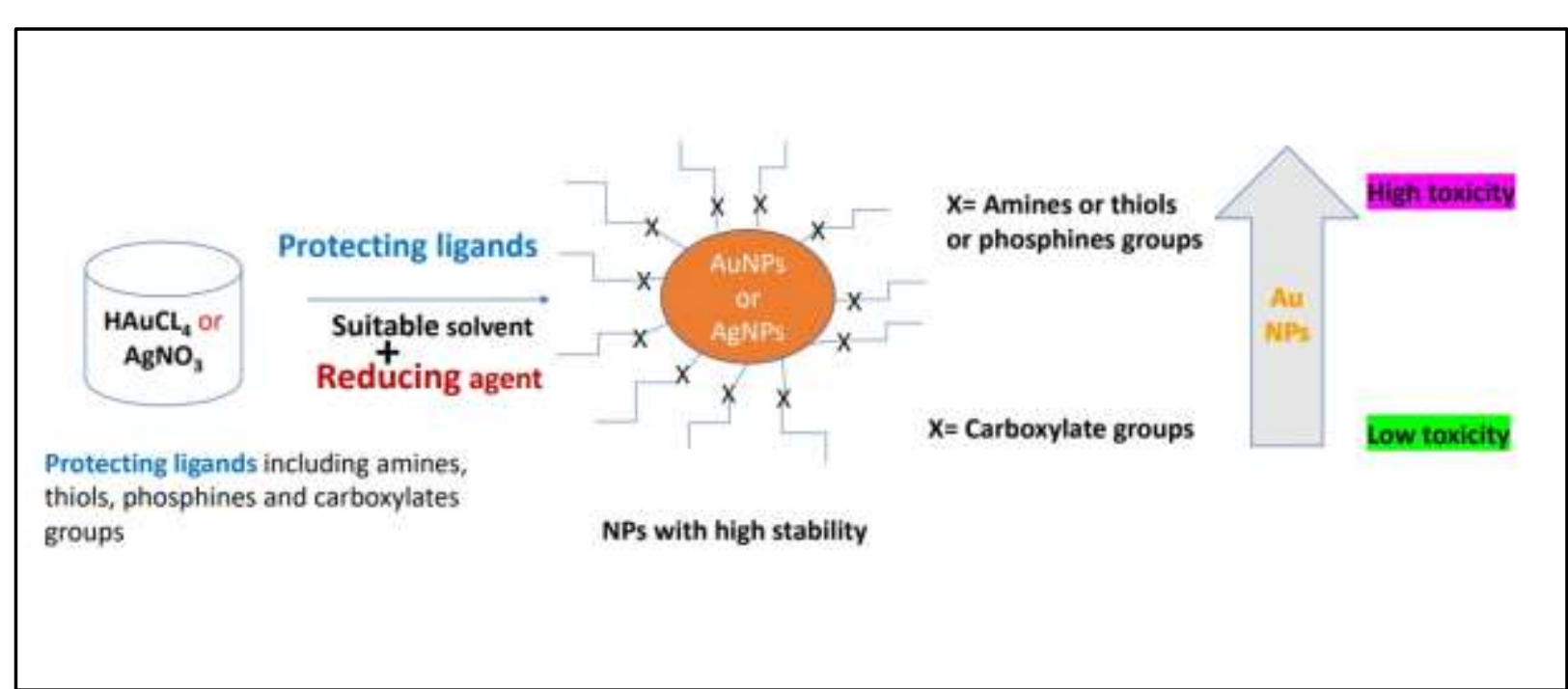

* Corresponding author's E-mail address: wanisa.mohammed@gmail.com; wani.Ahweelat@sebhau.edu.ly 


\section{Introduction}

Metal nanoparticles, such as Au (gold) and Ag (silver) nanoparticles (NPs), have been widely investigated over the past two decades due to their unique optical and chemical properties [1].

AuNPs have very high molar absorptivity in the visible region. Particle aggregation results in further colour changes of AuNP solutions due to mutually induce dipoles based on interparticle distance and aggregate size. This phenomenon can be applied to the various sensing systems [2-6]. Assembly of $\mathrm{Au}$ and Ag NP monolayers is one of the ideal substrates for surfaced-enhanced Raman scattering (SERS) [7, 8].

For critical applications, the NP surface needs to be modified using biocompatible materials. For several years, NPs have been tested in the medical field to reduce the side effects of cancer drugs via reducing their dose [9]. It is difficult to insure the absorption of a high dose of the drug at the desired site while preventing side effects (including drug toxicity) and using traditional antibiotics without a carrier. NP-based antimicrobial drug delivery systems can deliver the drug to the desired place, reducing the side effects [10]. There are several ways for synthesizing NPs that are environmentally friendly, low toxic, and not cost-effective. For example, iron oxide NPs (10-15 $\mathrm{nm}$ ) have been successfully prepared by Lactobacillus Fermantom cytoplasmic extract without using any stabilising agent or chemical reduction which indicates their potential future use in bioapplications [11].

Colloidal NPs have different applications, depending on their size and shape. For instance, by reducing the size, many changes were observed in physical and chemical reactions, such as increased surface/volume ratio and low melting temperature of AuNPs along with increasing size [10-12]. Many metal NPs such as silver have been used as therapeutics due to their antimicrobial properties.

AgNPs have been widely used due to their quantum size effect and large superficial area which is led to unique properties possessed by nanostructures. In biology and biochemistry, AgNPs have attracted a great deal of attention from the researchers. Over the last few years, scientists were established an important antimicrobial effect of AgNPs to fight infections and diseases [14]. For instance, AgNPs showed significant inhibitory effects against the microbial pathogens, and are widely used as antimicrobial agents in a diverse range of consumer products, including air sanitiser sprays, socks, pillows, slippers, respirators, and wet wipes. In addition, AgNPs exhibitd many interesting properties as size of AgNPs are decreased. The electromagnetic or physicochemical properties of AgNPs are quite different from the bulk material [13].

Over and above, zinc oxide nanoparticles (ZnO NPS) have gained considerable attention because of their electronic, unique antibacterial, antifungal, photochemistry, homogeneous catalysts, heterogeneous catalysts and ultraviolet (UV) characteristics $[15,16]$. ZnO NPs with bidentate schiff base ligands have illustrated notable biological properties [17]. Also, it was found that, the CFZ MNPs (Zinc-Doped Cobalt Ferrite Magnetic Nanoparticles) can float easily on the surface of polluted water and predicted to be as a good material for oil absorption and decreasing the environmental pollution [18].

Antibacterial activity of ZNO powder was found to be much weaker than ZnO NPs [19]. This can simply be explained as smaller particles that have a larger surface to volume ratio, producing a more effective means of antibacterial activity. The $\mathrm{ZnO} \mathrm{NP}$ surface is illustrated to be an effective inhibitor of bacterial growth [20]. Regarding the antibacterial activity of $\mathrm{ZnO}$ tested on both Gram positive and Gram negative bacteria, the 
effect was more pronounced with Gram positive than Gram negative bacteria. ZnO NPs also exhibited a preferential ability to kill HL60 cancer cells compared to the healthy peripheral blood mononuclear cells [15-21].

This review summarizes and discusses the proposed mechanisms of antibacterial action of different metal and metal oxide NPs. In addition, it discusses their bio-applications such as involvement in the production of reactive oxygen species (ROS), biomolecule interaction, and membrane interaction. General approaches of modifying the surface of noble metal nanoparticles and metal oxide nanoparticles by organic ligands will be outlined, as well. Finally, catalytic activity, stability of NPs and their toxicity will be discussed [15, 21, and 22].

\section{Fabricating NPS}

Nanoscience covers three main scientific areas including, chemical, physical, and biological sciences. The main goal of nanotechnology is to produce new materials that possess the size between 1 and $100 \mathrm{~nm}$ with special properties [23]. Utilizing of NPs with various sizes, shape, and structures which made it possible to manufacture the biocompatible and functional devices with special function in chemical, physical, and biological aspects [24-26].

\section{Synthesis of NPS}

The choice of method for preparing the composition of NPs depends on several factors including (1) the size of the NPs required, (2) the essential properties of the drug (for example, water solubility and stability), (3) surface characteristics, (4) degree of biocompatibility, biodegradability, and toxicity, (5) the release of the desired drug, and (6) the final antibody generator [23-27].

There are two general processes involved in the synthesis of NPs, including "bottom-up" and "top-down". In the bottom up method, the atoms are assembled to produce nanostructures. While, the opposite approach, the top down method, also identified as the physical method, where material is removed from the bulk material via grinding, chemical methods, or solid volatilisation is followed by steam condensation, leaving only the required nano-structures [1, 28]. Chemical methods such as reducing the metal salts in the solution are the most appropriate ways to control the size of the particles, modifying the chemical composition of the surface [1].

Both methods have essential advantages and can be applied in either gas, supercritical fluids, liquid, solid states, or in vacuum. Most of the producers have the ability to control one or more of the following aspects of the NPs including: a) size and shape NP, b) size distribution, c) particle structure, and d) particle agglomeration. An important aspect of both approaches is the stabilisation of the particles to avoid any aggregation $[1,27,29$, and 30].

The modern manufacturing techniques of hybrid nanoparticles have achieved controlled self-organisation of building blocks based on metal NPs. Fabrication of NP was focused on the use of building blocks for additional assemblies. Figure 1 illustrates several self-assembly patterns of metal nanoparticles $[1,30]$. 
Figure 1. Synthesis of metal nanoparticlebased building blocks and their use in bottom-up nanoscale manufacturing [1]

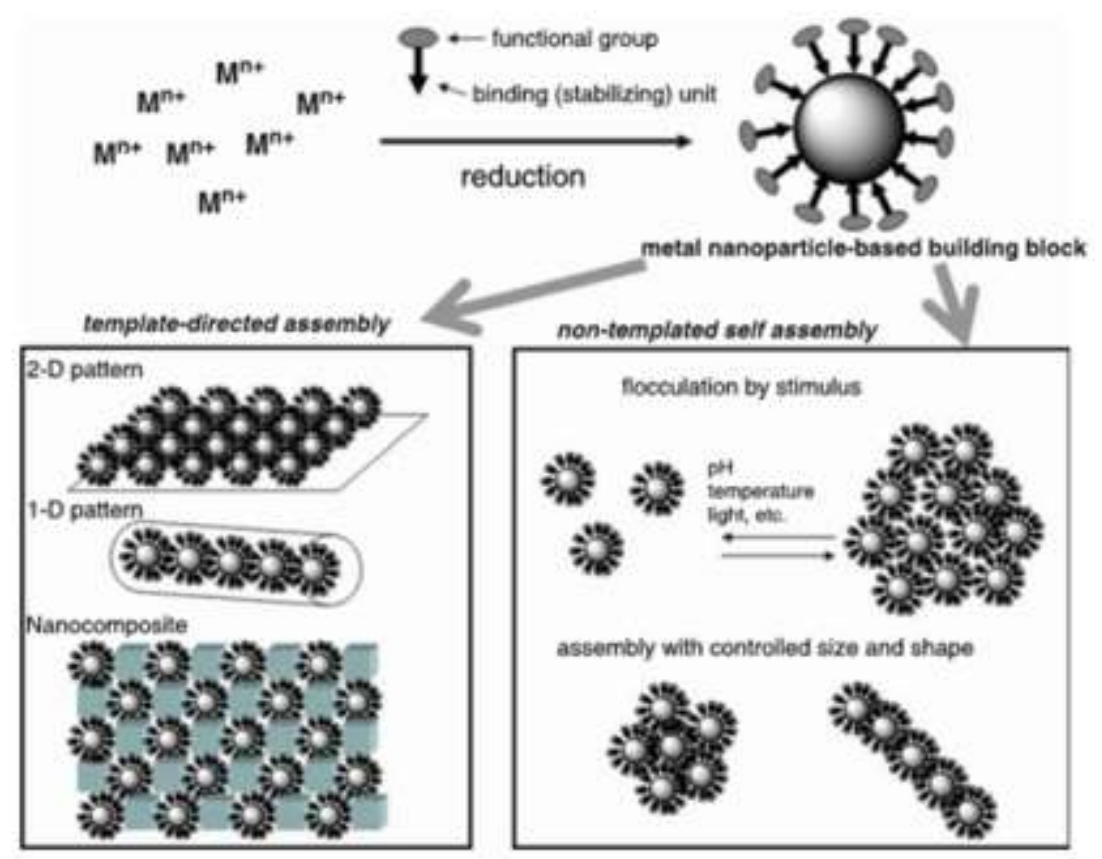

Fabrication of Nanoparticle-based Building Blocks

To exploit the properties of NPs for future device fabrication through a "bottom-up method," the fundamental challenge is the design of the NP-based building blocks. From a synthetic chemical viewpoint, these metal NPs are to be functionalized with a wide range of organic moieties using simple chemical process. The most significant condition for this purpose is the frequent isolation and redissolving in common solvents, then handling and characterization of the properties of the molecules. Metal nanoparticles can be stabilized by solvents or ions. Although they are ultimately useful for catalysis, they tend to irreversibly aggregate over time or extract from the solvent. To prevent the aggregation, metal NPs are protected by polymers and different ligands which have coordination properties for the metal surfaces that are generally prepared as colloidal forms via reduction of metal ions by $\mathrm{NaBH}_{4}$ in the presence of polymeric stabilisers such as poly(vinyl alcohol), poly(vinylpyrrolidone), and poly(vinyl ether) ( Figure 2). Stabilisation of metal NPs by ligands can enable further use, control solubility characterisation, and ease their analysis [1, 31, and 32].

\section{Stabilisation Methods of NPs}

Nanoparticles surface functionalisation plays a significant role in cellular uptake and producing cellular responses [33]. It is known that NPs have ability to form stable bonds with ligands contain sulphur atoms such as sulphides and thiols [34, 35]. These ligands have a strong affinity toward the noble metals and tend to protect AuNPs through forming well-ordered, self-assembled monolayers (SAMs), which leads to good dispersion, facilitate characterisation, and high stability. Without using protecting ligands, bare AuNPs tend to aggregate in solution as a result of losing electrostatic repulsion. So, protective coating ligand is necessary during synthesis to keep NPs stable [34-36].

\section{Stabilisation by Bipyridyl Ligands}

Nanoparticles stabilized by bipyridyl derivatives were described as a simple method to protect colloidal NPs with these ligands (Figure 3) [1, 37-40]. These ligands were also used to stabilize different kinds of metal NPs. For instance, platinum NPs have 
stabilised by reduction of Palladium (II) acetate in acetic acid solution by atom hydrogen at room temperature with 2,2bipyridine or 1,10 phenanthroline, followed by $\mathrm{O}_{2}$ treatment $[36,40]$.

\section{Stabilised by Phosphine Ligands}

Phosphine ligand derivatives have been widely utilized to stabilize NPs. For instance, colloids AuNPs were stabilized by phosphines when $\mathrm{HAuCl}_{4}$ was reduced by trisodium citrate and treated with excess of triphenylphosphine, which can be isolated via concentration of dilute solutions and addition of ethanol [37].

As the phosphine is easily oxidized in air, the synthesis should be carried out in an inert atmosphere. The phosphine-stabilised AuNPs undergo rapid exchange of capping ligand phosphine with dissociated and added phosphine in dichloromethane solvent at room temperature [36].
Figure 2. Preparation of metal NPs by using $\mathrm{NaBH}_{4}$ as reducing agent in the presence of cationic surfactants (a) and polymers (b) [1]

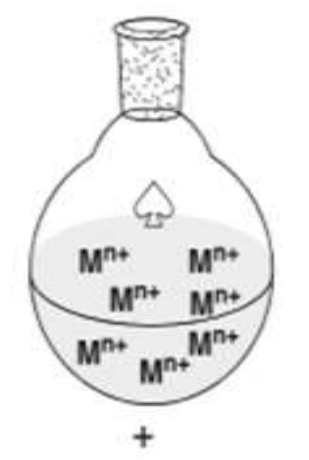

Reducing agents

$\mathrm{NaBH}_{4}$ $\mathrm{EtoH}$
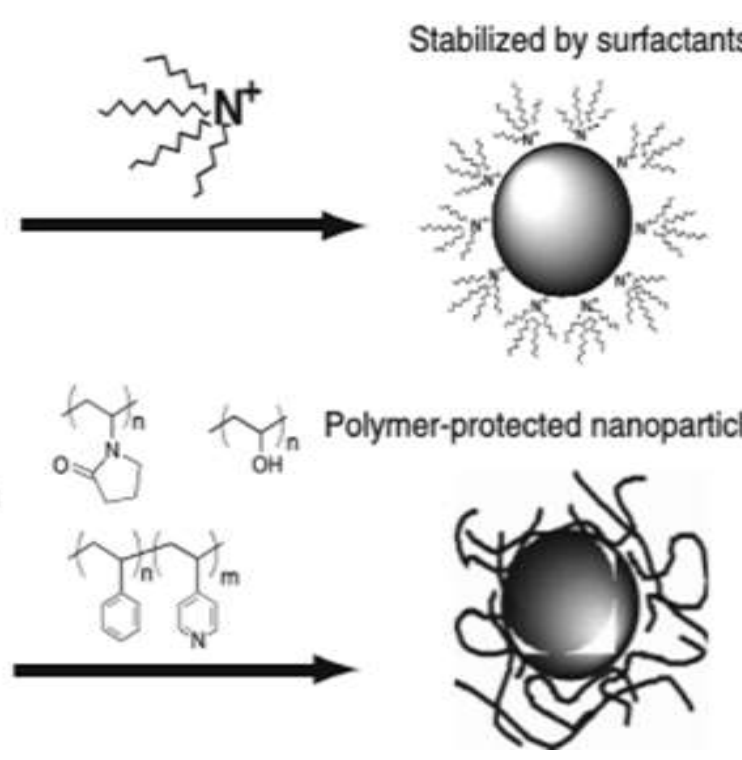

Figure 3. Examples for ligands that are used to stabilise metal NPs including amine, phosphine, and bipyridyl ligands [1]
Stabilized by Amines and Phosphines
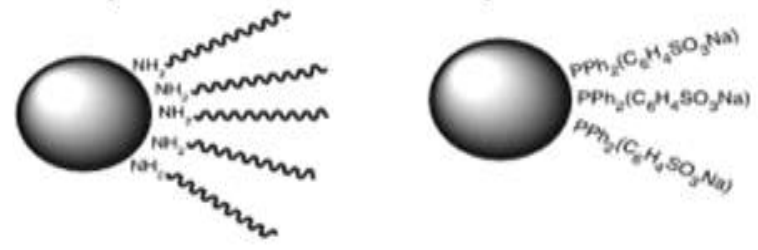

Stabilized by Bipyridyl Ligands

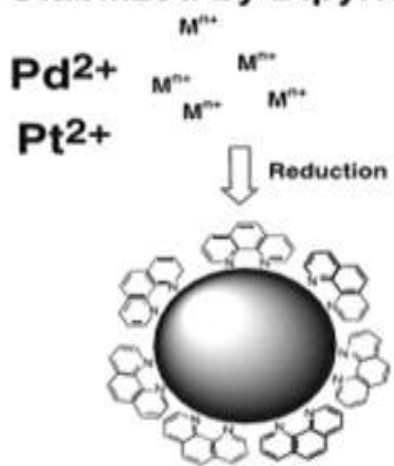


Ligand exchange reactions of triphenylphosphine-stabilised NPs with $\omega$ functionalised thiols provide a versatile approach to functionalised, $1.5 \mathrm{~nm}$ AuNPs from a single precursor [41].

It was found that, in all cases the materials produced with the phosphine ligands stabilized NPs displayed the similar properties (such as size, stability, and solubility) as those derived from the Schmid Method [42]. Likewise, AuNPs were successfully synthesized using trioctylphosphine and tricyclohexylphosphine as stabilized ligands that prepared under the same conditions. The ability of easily prepare larger amounts of phosphine stabilised NPs in a single step with sodium borohydride $\left(\mathrm{NaBH}_{4}\right)$ as reducing agent can be encouraging to use of these NPs in a broader range of applications [42].

Furthermore, one advantage of phosphine derivatives such as triphenylphosphine (TPP) stabilised NPs like gold ( $\mathrm{PPh}_{3}$-AuNPs) is that the phosphine ligand as a stabilizer, enabling easy replacement by other ligands such as thiols, amines, and other phosphines to control the material properties. It is known that TPP-AuNPs have important applications in different fields including, catalysis, microelectronics, sensors, drug delivery, biological imaging, and immuno-assays. For all of these significant applications, synthesis of stable, small, and uniform NPs is considered the initial challenge [37-41].

In addition, tris (3-sulfonatophenyl) phosphine is used to functionalised $\mathrm{Au}$ and $\mathrm{Ag}$ NPs. The resulting NPs of AgNPs were diameter 15-25 nm, AuNPs were 5-6 nm, Ag$\mathrm{Au}$ NP (50:50; was 10-12 $\mathrm{nm}$ ) and they were easily dispersable in aqueous media. Biologic effect of these NPs toward human mesenchymal stem cells (hMSC) was different. For example, AgNPs showed an important concentration dependent influence on the viability of hMSC. However, AuNPs exhibited only a small effect after 7 days on the viability of hMSC. In contrast, Ag-Au NPs had no important influence on the viability of hMSC [43].

\section{Stabilised NPs by Thiol Ligands}

The ability of modified NPs, such as gold NPs with various organic thiolate ligands make them versatile systems and open variety of possibilities for their use as drug delivery systems, and bio-applications [33]. Capping agents used with strong affinity for NP are a common approach, which cause the preparation of NPs with good size dispersion in water. In some case, extra steps are required to transfer the NPs into water. However, the transfer phase from aqueous to organic phase is often not easy, due to the poor water solubility of the protecting ligands used to stabilize the NPs.

Mercapto groups (RSH) have been also used as stabilisers of metal NPs, particularly for gold. Preparation of colloidal AuNP solutions was carried out following the principals involved in the method developed by Brust and Schifrin method, a two-phase system. For instance, the dodecane-thiolate ligand used for stabilizing the AuNPs (Figure 4), where $\mathrm{AuCl}_{4}{ }^{-}$is transferred from aqueous phase to organic phase (toluene) and using tetraoctylammonium bromide as a phasetransfer reagent and then reduced by $\mathrm{NaBH}_{4}$, yielded AuNPs having average range of 2-8 $\mathrm{nm}$. The size of the resulting AuNPs can be decreased by increasing the thiol/ $/ \mathrm{HAuCl}_{4}$ ratio [28-33]. These monolayer-protected AuNPs are repeatedly isolated and redissolved in common organic solvents such as toluene, dichloromethane, and hexane without aggregation or fusion. Monolayerprotected silver and $\mathrm{ZnO}$ nanoparticles can also be prepared by using the same protocol [44-47].

The alkanethiolate monolayer-protected NPs are insoluble in aqueous solution, so it was important to find alternative solvent to water as mentioned in the literature [33], and 
it was dimethyl sulfoxide (DMSO). So, according to the FDA, DMSO is only organic solvent approved by USA Food and Drug Administration (FDA) for drug carrier tests, which indicates its potential future use in biomedical applications [33].

The lack of poor stability of alkanethiolates in water can be overcome by the use of ligands exchange of these monolayer protected NPs with various functional thiols in the organic solvent as revealed in Figure 5. Where the place-exchanged NPs bearing polar $\omega$ - functionalities can be dissolved in several polar solvents $[1,28]$.

\section{Stabilised NP by Amine Ligands}

Figure 4. Preparation of gold nanoparticles protected with monolayer of alkanethiols (the BrustSchiffrin method) by twophase solvent system [28]

Figure 5. General scheme for the place-exchange reaction between alkanethiol monolayerprotected gold nanoparticles and various functional thiols. Where in the bottom scheme, $x$ and $m$ are the number of new and original ligands,
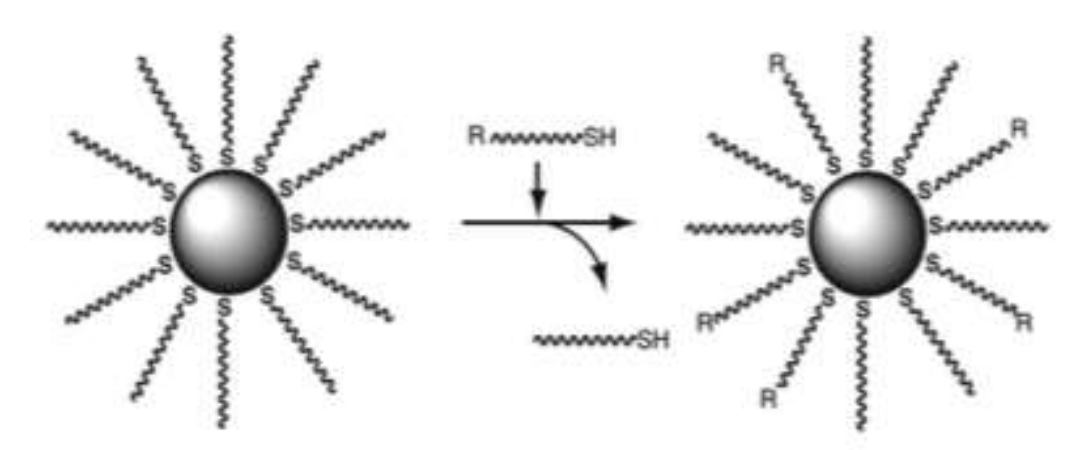

respectively [1]

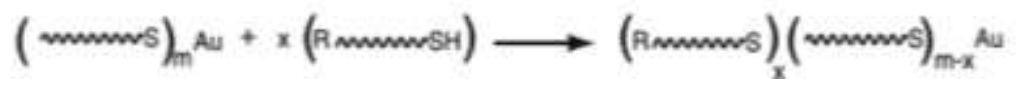

Stabilization of metal NPs (such as Au, Ag, $\mathrm{Cu}$, and Pt NPs) by using suitable organic ligands such as amine derivatives is widely used. Alkyl amines and amino acid lysine have been used as protective ligands. However, their stability for AuNPs are moderate in comparison to both thiol and phosphine ligands [48].

The main difference between thiol and amine functions is the stability of the metal NPs. For instance, metal NPs have bigger sizes when functionalized by amine ligands rather than thiol ligands, due to weak affinity between metal NPs and amines which led to weakly bound and chemically unstable monolayers on bulk NP surfaces. While, both of thiol and amine can be complementary.
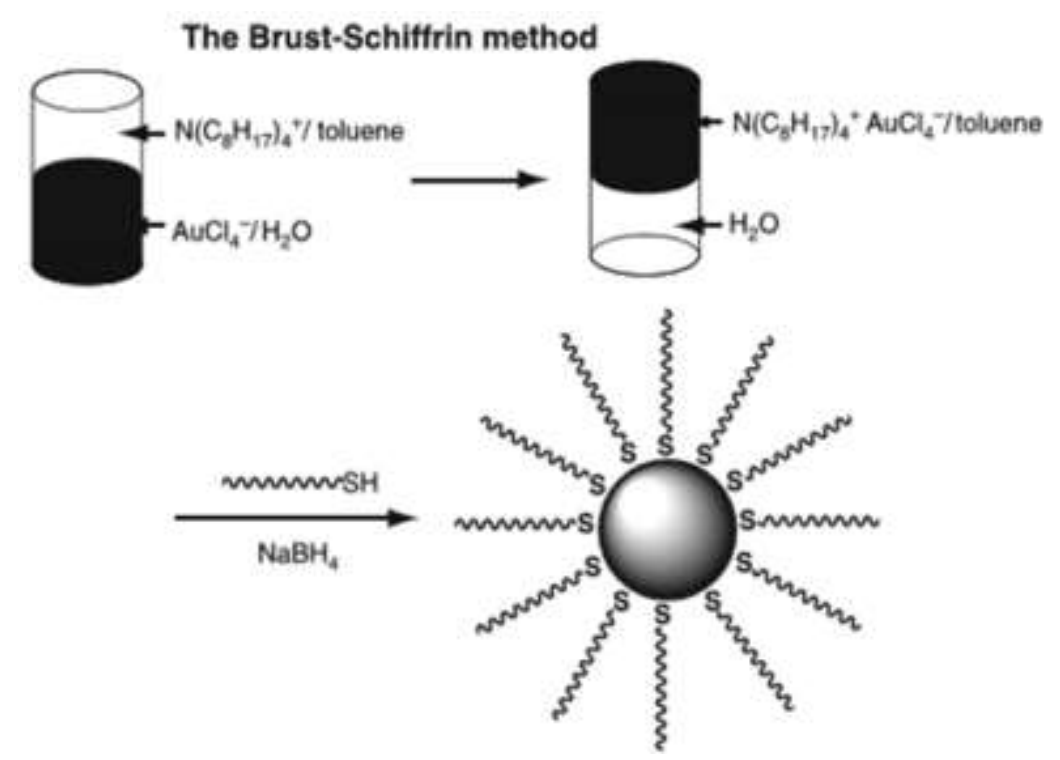
Peptides are linked to AgNPs through both the thiol and amine functions [49]. Gold and silver nanoparticles protected by aminoligands can produce Au with 6-21 nm and Ag NPs with 8-32 nm [1].

Metal oxide NPs also functionalized by different ligands, but the main compounds used for modifying metal oxide NPs are phosphonates and carboxylate ligands which in especial fatty acids are usually used for functionalising metal oxide NPs [49]. However, silane ligands are the most often used modifiers for metal oxide NP surfaces, such as alkoxysilane, hydrogeno-silane, or chlorosilane reagents. One of the main advantages of silanes is easy to synthesis. They also can bear numerous functionalities, such as amino, carboxylic acid, cyano, and epoxy groups [49].

According to information published in the literature, the functionalised amines ligand AuNPs produced good results, leading to monodisperse, AuNPs (Figure 6) with diameters between 1.3-4.2 nm. Also, they noted the size of functionalised NPs reduced with increasing of length of the amine ligand chain. This may be rationalised through the longer chains hindering access to the surface of the NPs more effectively in comparison to the shorter ones limiting NP growth. In addition, it explored the effect of reaction time on monodispersity and size of NPs. For instance, increasing the reaction times caused to a growth in the size of the AuNPs stabilised by octadecyl-amine ligand. Consequently, the size of the NPs can also be controlled via changing time of the reaction, while kept high monodispersity [50].

According to Noorizadeh et al. [51] reported that, amino ligands such as (3aminopropyl)-triethoxysilane were used to functionalize iron oxide $\mathrm{NP} / \mathrm{SiO}_{2}-\mathrm{NH}_{2}$ for selective removal of arsenic (which is an extremely toxic metalloid for human, plants and animals) in water is evaluated. It was shown that the adsorption of arsenic on the NP was based on both $\mathrm{pH}$ and contact time. $\mathrm{On}$ the same hand, $\mathrm{SiO}_{2}-\mathrm{NH}_{2}$ used as protected ligands, where increased the stability of NP and prevented the agglomeration of NPs, and produced a high tunable surface area, which allowed to high binding ability and excellent adsorption properties for arsenic (III). This adsorbent was stable, low-cost, and environmentally friendly and showed potential application for arsenic (III) removal [51].

Small size of AgNPs (6 $\mathrm{nm}$ ) have been synthesised through the hydrogenolysis of Ag-amidinate complex in the presence of hexadecyl-amine (HAD) as a stabilised ligand which is strongly coordinated to the Ag NP surface and it is necessary to stabilise the NP and avoid uncontrolled aggregation [52].

Figure 6. Synthesis of gold nanoparticles stabilised by amine derivatives ligands [50]

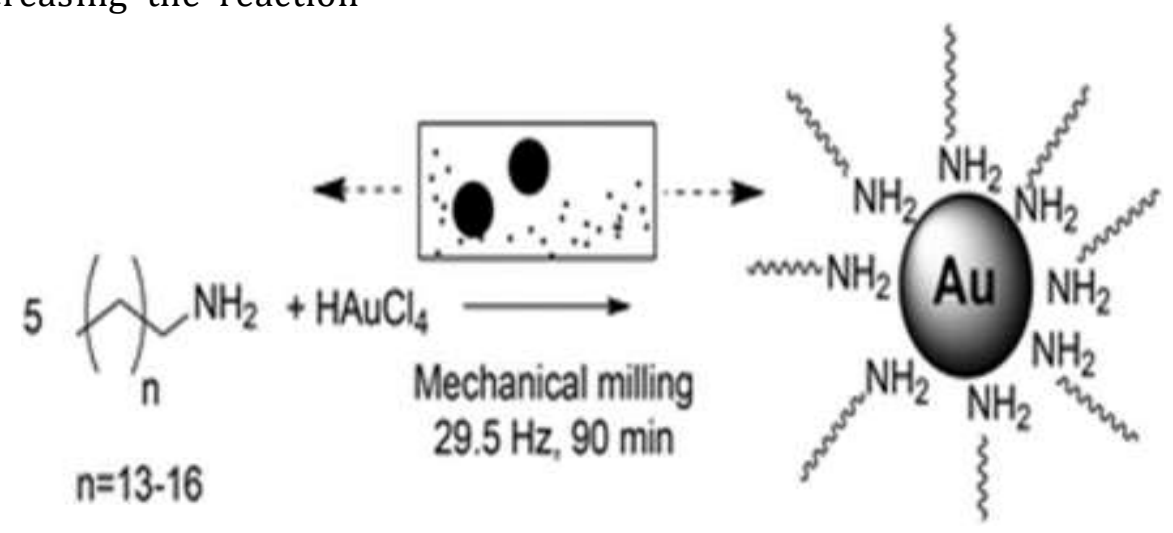


used for increasing the stability and facility dispersion of the metal NPs. These agents may potentially have a direct effect on the toxicity of the NPs as result of their ability to reduce the NP agglomeration [53-55]. It was found that, citrate- and chitosan-capped AgNPs can kill the bacteria, as a result of an accelerated generation of Ag+ from these NPs [53]. In addition, the capping agent chitosan have shown antibacterial activities against E. coli, but in concentrations $>200 \mathrm{ppm}$, proposing that the antibacterial activities of chitosancapped Ag NPs are not connected to this polysaccharide [23]. Moreover, protecting ligands can increase NP anti-bacterial activity on the bacteria due to their uniform size and excellent stability [56].

\section{Size, Shape, and Charge Characteristics of NPs}

Synthesis of small and monodisperse NPs is considered to be a major challenge in nanotechnology research. Normally, smaller NPs have higher antibacterial activity $[23,57]$ than big sizes. In addition, $1.4 \mathrm{~nm}$ AuNPs capped with triphenyl phosphine mono sulfonate were toxic in cancer cells and more toxicity from AuNPs with $15 \mathrm{~nm}$ in diameter [58]. Smaller NPs tend to be more toxic compared with that of the large NPs, as the small NPs have larger surface area to volume ratio in comparison with larger NPs which can significantly increase the production of ROS, that consequently can damage, disrupted metabolic activity and inactivate essential biomolecules, such as DNA, proteins, and lipids. Moreover, ROS production was induced generally through the introduction of both metal oxide and positively charged metal nanoparticles in the cell, such as metal oxides and silver NPs [59].

Penetration of NPs into tumours is influenced by their size, shape, and surface charge. Small NPs enhance tissue penetration but those smaller than $10 \mathrm{~nm}$ are cleaned by renal excretion and phagocytosis. NPs about $100 \mathrm{~nm}$ exhibit enhanced circulation half-life compared to smaller or larger particles. Polystyrene NPs of $20 \mathrm{~nm}$ and $40 \mathrm{~nm}$ penetrated the tumour and distributed homogeneously whereas 100 and $200 \mathrm{~nm}$ particles presented restricted penetration [60].

However, some literatures have shown that larger NPs are more effective, illustrating that size alone is not the most important factor of their toxicity $[61,62]$. There are other factors that can be considered such as the formulation process, the bacterial defence mechanism, the environment, and the physical characteristics of the NP. NPs are supposed to be able to participate in subcellular reactions because their size is similar to biological molecules [40]. NPs have different properties due to their size and surface chemistry, so that have demonstrated the ability to inhibit bacterial growth and thus have been used as a tool to combat infectious diseases [15, 23]. In addition, Mukha et al., [63] showed that Ag NPs of $<10 \mathrm{~nm}$ pass through the pores in the cell membrane, allowing penetration of microbial cells. Therefore, high antimicrobial activity of the AgNPs synthesised is provided by a major parameter such as size [63].

For NPs, the most common shape is spherical, although other shapes such sheets, plates, rods, tubes, cubes, and triangles have also been reported. Nano-cubes and rods (like $\mathrm{CeO}_{2}$-NPs) seem to be more effective than other shapes, maybe due to the exposed surfaces and to the oxidation levels of the metals [64]. This interpretation was supported by the analysis of exposed crystal faces, indicating that the less stable surface requires less energy to form oxygen-free functions, and linking the microbial activity of the NPs with the stability of the surfaces [44].

The interaction between shape and cellspecificity has been critical for cellular uptake. Herceptin-coated nanorods exhibited increased internalisation than the spherical or disk-shaped NPs in BT-474 breast cancer cells 
[60]. Even between NPs with identical surface areas, the shape is significant because the surfaces with high atom density can increase the reactivity [23].

\section{Antimicrobial Activity of NPs}

Bacterial infection is the major cause of chronic diseases and mortality. Antibiotics have been used to treat bacterial infections. However, super-bacteria are mostly resistant to all antibiotics. Present antibiotics are not effective against bacterial infections as result to their low intracellular retention and poor permeability. Recently antimicrobial drug resistance is considered one of the most serious threats to global health. For this reason, many researchers have tried to develop new, effective antimicrobial reagents at low cost, fewer side effects, and free of resistance. Most antibiotic resistance mechanisms are not related to natural antibiotics because the antibiotic method of action is direct contact with the bacterial cell wall, without the need to penetrate the cell; this raises the hope that neuroprotective agents are less likely to enhance resistance in bacteria than antibiotics [15,23, and 33].

In addition, it is found that NPs are more effective when combined with antibiotics drugs because it is easier for the NPs to enter the cells compared with antibiotic drugs alone. And that leads antibiotics to enter the cells very easily [65]. It should be noted that NPs did not appear to undergo morphological change with the addition of antibiotics [66]. Several studies have shown that NPs toxicity is significantly higher for many bacterial species $[10,67]$, so nanotechnology may be particularly useful in treating bacterial infections in future.

NPs have illustrated widely antibacterial properties against both Gram-positive and Gram-negative bacteria. For instance, $\mathrm{ZnO}$ NPs have found to inhibit Staphylococcus aureus, while Ag NPs exhibited concentrationdependent antibacterial activity against
Pseudomonas aeruginosa and Escherichia coli [10].

\section{Mechanism of Penetration of NPs}

Penetration of NPs across the endothelium of the blood vessels into target tissues is not easy in nano-treatment [60]. In normal healthy conditions, NPs cannot cross the endothelium of blood capillaries. While, in certain pathological conditions, such as cancer or inflammation, endothelial cells lose the cellular integrity as a result of activation of proinflammatory cytokines and the gap between the endothelial cells is increased. Consequently, NPs can extravasate from the vascular system to the wanted tumour cells via the abnormal endothelial gaps [60].

Based on current researches, the mechanism of antibacterial effects for NPs are as following: firstly, disrupt bacterial cell membrane; secondly, generation of ROS, thirdly, penetrating the bacterial cell membrane, and finally induction of antibacterial effects within cells, including interactions with proteins and DNA [10,15, and 59].

NPs introduce ROS into bacteria by diffusion. For example, Zhang et al [68] was examined the ROS generation mechanism of gold $(\mathrm{Au})$, silver $(\mathrm{Ag})$, nickel $(\mathrm{Ni})$, and silicon (Si) NPs in aqueous suspension under UV irradiation (365 $\mathrm{nm}$ ). The result presented that Ag NPs showed good antibacterial activity and generated hydroxyl radicals and superoxide, while Au NPs, Ni NPs, and Si NPs generated only singlet oxygen, which entered cells and producing an antibacterial effect [68].

It is well-known that Ag NPs and ZnO NPs have ability to penetrate the P. aeruginosa (as a Gram-negative bacteria) and $S$. aureus cells (as a Gram-positive bacteria). However, the Nickel Oxide NPs (NiO-NPs) did not exhibit well interaction with $S$. aureus at $2 \mathrm{~h}$, but it seemed to show more affinity toward $P$. aeruginosa. The affinity of NPs for bacterial 
membranes occurred as results to most of the membranes are negatively charged. So, it is probable that the positively charged NPs might have more affinity for the bacteria membranes [69].

On the one hand, the differences in bacterial cell walls, will lead to different results when testing with NPs. For example, Gram negative bacteria have thinner cell wall comparing to Gram positive bacteria. Also, it is reported that E. coli ATCC 8739 was more resistant against nano silver than Gram positive Pseudomonas aeruginosa ATCC 9027. It has also dependent on the concentration and size of NPs. For instance, AgNPs with size of 11-36 nm were reported to be most effective against bacteria through direct interaction with bacterial cells. While the interaction of Pseudomonas aeruginosa ATCC 9027 with AgNPs was shape-dependent particles and showed the highest activity [70, 71]. In the same way, AuNPs found to be more effect on Gram Negative (such as E. coli and Pseudomonas aeruginosa) than Gram Positive (Staphylococcus aureus, Micrococcus luteus), which explained to their easier incorporation into gram negative bacteria [70].

It is demonstrated that the particle size of $\mathrm{NP}(\mathrm{Ag}, \mathrm{Au}) \sim 2 \mathrm{~nm}$ that can penetrate the cell and showed direct interaction with many bacterial strains, the stronger antimicrobial capability of NP is due to the high surface to volume ratio of NPs, which can be leading to generate more active oxygen species per unit weight. The activity of the oxygen species is perhaps executed to the cell membrane subsequently it also produced serious haemolysis on rat red blood cells.

Several mechanisms are proposed in the literatures [72]. It is believed that the interaction between negatively charged bacterial membranes and positively charged $\mathrm{Ag}$ ions plays a main role in causing the membrane rupture and cell lysis. It is also assumed that $\mathrm{Ag}^{+}$prevents DNA replication. Additional mechanism includes interacting with thiol groups of proteins, inactivating and blocking of respiratory enzymes [72]. As well as, $\mathrm{Ag}$ NPs share the mechanism with $\mathrm{Ag}^{+}$in binding thiol-group containing proteins that are abundant in the cell membrane, consequently leading to cell envelope abnormalities [72]. The Ag NPs adsorbed on outer membrane of cell wall of $S$. aureus and $S$. typhimurium are penetrated and accumulated in cells without causing any aggregation or making damage for neighbouring cytoplasm [73].

Moreover, AuNPs were used with a $5 \mathrm{~nm}$ size as an antimicrobial effect on $E$. coli and $S$. aureus bacteria species. It was described that about $90-95 \%$ of all bacterial colonies had decreased as result to AuNPs effective. Furthermore, the smallest NPs have the strongest antimicrobial effect compared to the larger sizes, which explained due to their ability to interact easily with bacterial cells, where bacteria have size about 50 to 5000 times bigger than NPs [74,75].

In addition, ZnO-NPs known to have high catalytic and high photochemical activities addition to its unique antibacterial and antifungal properties. When particle size is reduced to the nanometer range, then nanosized $\mathrm{ZnO}$ can interact with bacterial surface where it enters inside the cell, and subsequently damage bactericidal cells [76].

Interactions between these NPs and bacteria are generally toxic and have been benefited for antimicrobial applications as in the food industry. It is well known that modify of $\mathrm{ZnO}$-NPs surface area led to release of $\mathrm{Zn}^{2+}$ ions and enhance ROS production. For instance, the release of $\mathrm{Zn}^{2+}$ ions were a logical cause of ZnO toxicity against Saccharomyces cerevisiae bacteria where these bacteria are common in food processing which caused damage to the cell wall $[10,76]$.

\section{Catalytic comparison of $\mathrm{Au}, \mathrm{Ag}$, and $\mathrm{ZnO}$ NPs}

Intensive researches have been interested 
on the development of catalytic systems owing to their significance in synthetic organic chemistry. One of the most attractive synthetic strategies preferred by organic chemists is the use of heterogeneous catalyst in order to increase the efficiency of a varied range of organic synthesis. Heterogeneous catalysis is being used in the chemicals industry due to the wanted environmentally friendly production technology [77]. For example, nanoporous-isopolyoxomolybdate $\left(\mathrm{NH}_{4}\right)_{42}\left[\mathrm{MoVI}_{72} \mathrm{MoV}_{60} \mathrm{O}_{372}\left(\mathrm{CH}_{3} \mathrm{COO}\right)_{30}\left(\mathrm{H}_{2} \mathrm{O}\right)_{72}\right]$ is used in multicomponent reactions and was as a very important green catalyst because of its stability, non-toxicity, high solubility in water, ease of treatment, inexpensiveness, and high availability which allow this material to catalyse various transformations in the organic chemistry [78].

In addition, metal oxides constitute the largest family of catalyst in heterogeneous catalysis as a result of their base-acid and redox properties. For example, bulk Zinc oxide was employed as a heterogeneous catalyst for numerous organic transformations. Also, nano $\mathrm{ZnO}_{12}$ was used as heterogeneous catalyst and exhibited significant attention because of its inexpensive, non-toxic catalyst and has many environmental advantages such as recycling of the catalyst, minimum execution time, and waste minimisation. In general, the NPs are predicted to be very reactive due to their higher surface area and small sizes. The surface area of the catalyst is increased when size decreases to nano levels which are responsible for a strong catalytic activity [77]. Furthermore, it is notable that the reaction carried out by changing the size of the particles from NPs to bulk caused in a drop in the catalytic activity. It is remarkable to mention that the ZnO NP catalyst catalyses the reaction in great yield within a shorter time of reaction than the corresponding bulk Zinc oxide [77]. The catalytic properties of metal NPs have produced great attention over the past decade [1].
For example, researches on interaction of alcohols with ZnO NP has revealed that $\mathrm{C}-\mathrm{O}$ bond of the alcohol is cleaved on surface of zinc NP giving hydrocarbons and the oxide species on the NP surface [77]. It is known that the catalysts contain an oxide as an active phase are widely applied in the chemical, environmental pollution control and petrochemical industries contain an oxide [16, 79].

Current emphasis of researchers on green chemistry highlights the requirement for developing catalytic reactions in biocompatible solvents. One of important requirements for achieving this goal is to explore alternative reaction conditions and reaction solvents to achieve the desired chemical transformation with reducing unwanted products, waste generation in addition to eliminating the use of volatile and toxic organic solvents [80]. For instance, green synthesis of Ag NPs has been successfully synthesised by using green sources like microorganisms, plant extracts, and some biopolymers without producing of hazardous wastes and this NP found to be a more economical and efficient biosynthesis procedure. Moreover, the catalytic activities of the synthesised, Ag-based recyclable nanocatalysts using several plant extracts in different chemical reactions such as oxidation, reduction and have produced good results [81].

It was observed that the $\mathrm{Au}$ NPs synthesized in Ionic Liquid (IL) catalysed the condensation between aldehydes and active methylene compounds and generated products with excellent yields in short time. This methodology has several advantages such as avoiding of expensive reagents and very high temperatures also it is considered to be very safe for the environment [80].

Further, it is well documented that Ag NPs in ionic liquid presented the best activity for the formation of disulphides. Ag NPs have been found to be recyclable and reused for 
other reaction, which makes it inexpensive or economical [80]. Silver-based catalysts are particular interest because of a number of valuable properties of metals. These including the ability to activate molecular oxygen, and the homogeneous distribution of metal $\mathrm{NP} /$ clusters on different supports such as $\mathrm{SiO}_{2}, \mathrm{CeO}_{2}, \mathrm{Si}_{3} \mathrm{~N}_{4}$, and $\mathrm{TiO}_{2}$. These features became the basis for the application of Agbased catalysts in methanol oxidation in the industrial gaseous phase to form formaldehyde and oxidation of ethylene glycol to glyoxal. Recent trends in the development of Ag-based catalysts for selective oxidation of alcohol have been summarised in both gas and liquid phase processes as well as in photocatalytic applications [82].

From the literature, AgNPs are known to be efficient catalysts for the organic dyes reduction [83] and illustrated excellent catalytic activity in reduction of 4-nitrophenol to 4-aminophenol via using $\mathrm{NaBH}_{4}$ as reducing agent. It is found that highly antimicrobial and catalytic active AgNPs can be produced using phenol or phenolic compound degraded by microorganisms and further progress in the process may result to use these AgNPs in industries and water purification [84]. As well, catalytic reduction of 4-nitrophenol was sensitive to the amount of Ag NPs present in the composite. It is also noted that when the

Figure 7. AuNPs prepared by $4-\mathrm{NP}$ reduced by $\mathrm{NaBH}_{4}$ in aqueous solution [85]

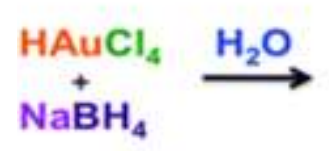

concentration of the catalyst was low there was important induction period in the reaction, whereas the reaction started directly when the concentration was quite high [83].

Moreover, $3 \mathrm{~nm}$ AuNPs are prepared by reduction of $\mathrm{HAuCl}_{4}$ by $\mathrm{NaBH}_{4}$ in water under ambient conditions which is very efficiently catalyse 4-nitrophenol reduction to 4aminophenol (Figure 7) [85].

The catalytic activities of freshly synthesised $\mathrm{Au}$ and Ag NPs are known to be very sensitive to the nature of the $\mathrm{Au}$ and $\mathrm{Ag}$ NPs surface. For example, $\mathrm{Au}$ and AgNPs under $\mathrm{N}_{2}$ have been compared for the reduction of 4-nitrophenol to 4-aminophenol, and it is found that AgNPs were $3 \mathrm{~nm}$, and AuNPs were $4.5 \mathrm{~nm}$ in the same condition. The increased catalytic activity is connected to the increased number of electrons on the $\mathrm{Au}$ and Ag atoms [86].

On the other hand, the agglomeration of metal NPs subsequent from insufficient stabilisation by ligands, which leads to the formation of larger-size and sharp of NPs then reducing their catalytic activity. For this reason, it is essential to use a protective ligand, and this can avoid any possible agglomeration of NP may occur, stabilise dispersed NPs, further protect NPs from oxidation [86].

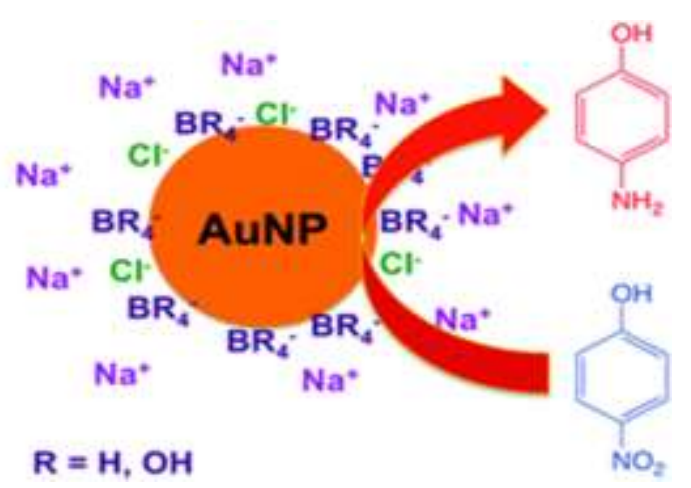

properties can be significantly improved by organising two types of metal NPs to produce intermetallic nanocomposites because of synergistic effects [1]. Even a physical mixture of two types of metal NPs illustrated high 
catalytic activity compared to the corresponding monometallic NPs. For instance, AuNPs functionalised by a carboxylic acid were combined with silica NPs that stabilised by a primary amine, acid-base chemistry followed by immediate charge pairing produces electrostatically bound associated colloid constructs $[1,87]$.

It was also shown that the size of the bimetallic NPs influenced the catalytic properties of the Au-Ag NPs. The reduction reaction rate for the smaller size of Au-Ag NPs catalysts was higher than the larger size of particles. The bimetallic Au-Ag NPs significantly improve the catalytic activity in comparison with the mono-metallic Au NPs [88].

In addition, it has also demonstrated that the $\mathrm{Au}$ NP-polyaniline composite on a resin bead can act as a catalyst for the oxidation of glucose with simultaneous colorimetric detection of the product. Several other researches have subsequently developed many synthetic schemes to produce metal NP-polymer composites for suitable applications. For instance, several metal NPpolymer composites have synthesised for catalysis with combination of thermosensitive activity as one of the major advantages. Nevertheless, incorporation of Ag NPs into polymer, particularly biopolymer, has not received a lot of attention, perhaps because of the experimental difficulty in the preparation of composites. The Ag NPs produced were small sizes generally below $5 \mathrm{~nm}$, with high catalytic activity [83].

According to X. Fang et al., [89] some biosynthesised NPs are typically used as heterogeneous catalysts in environmental remediation and displayed higher catalytic efficacy due to their enhanced biocompatibility, large specific surface areas and high stability. Compared with NPs produced by traditional methods, biosynthesised NPs have some unique properties and can be used without any side effects in areas such as chemical catalysis and degradation of organic pollutants. For example, some specific pollutants like dyes are removed by some biosynthesised NPs which play the role of catalysts. These dyes are known to be dangerous to almost all living organisms as result to their toxicity [89].

In addition, several industries, such as the food-processing, drug, widely use dyes which led to severe pollution in soil and water. Some conventional methods were used to remove the remaining dye in the industrial process, but the results were not satisfactory. The low efficiency of the removal remains the main problem for this approach. By contrast, biologically manufactured NPs show excellent catalytic performance due to their high surface area. It has also been shown that biosynthesised NPs are a viable alternative to the physical and chemical methods traditionally used in chemical catalysis, especially in the removal of organic compounds. However, there are still limitations in bio-synthesis of NPS: poor production, reduced control of product quality, and hard separation of NPs from the biological materials [89]. On the other hand, AgNPs synthesised using different physical and chemical techniques have shown promising applications in the catalytic degradation of organic dyes [90].

Based on the $\mathrm{pH}$ factor, AuNPs functionalised by carboxylate-modified poly( $N$-vinyl-2-pyrrolidone) have shown good abilities to be efficiently catalyse the aerobic oxidation of benzyl alcohol in aqueous solution under mild conditions and are easily separated from the reaction mixture via the reducing $\mathrm{pH}$ of solution, leading the NPs to precipitate [91]. Also, AuNPs with diameters < $10 \mathrm{~nm}$ were actively targeted as oxidation catalysts for toxic fumes such as $\mathrm{NO}$ and $\mathrm{CO}$ [92]. Due to its high stability than other metal nanoparticles, AuNPs have been used as well as catalysts in cyclisation reactions, rearrangements, addition to multiple $\mathrm{C}-\mathrm{C}$ bonds, and $\mathrm{C}-\mathrm{C}$ coupling reactions $[93,94]$. 


\section{Toxicity of NPs}

Currently, few researches have to date focused on the toxicity of NPs on the environment and human resources, toxicity of NPs can occur mostly due to particle size or charge $[10,95]$. AgNPs are used to produce antibacterial products like clothing and food containers. The potential problem is the release of AgNPs. For example AgNPs are released gradually in the environment where the products are used, washed and then disposed in the end. According to literature [10], AgNPs can cause hazardous changes in soil bacteria populations, lead to reduce the population of bacteria that fix atmospheric nitrogen. These bacteria convert nitrogen of the atmosphere into ammonia and other nitrogen compounds that can be used by plants. Where the soil becomes infertile without these bacteria [10]. In contrast, Ag NPs in most studies are reported to be less toxicity than silver ions. For instance, the toxicity of silver ions were described to be about 3.4 times higher than the AgNPs [72].

In addition, AgNPs can release silver ions as mentioned early, which can be toxic. As in these studies reported in the literature, the use of AgNPs for drug delivery for human should be limited. AuNPs found to be particularly effective for photoablation therapy whereas AgNPs would better to be used for controlling bacterial infections. because using outside these fields may require technical advances in order to reduce the negative toxic effects of these agent on animals [92]. According to reported studies in the literature, the toxicity of some NPs suggested to be only partially related to their chemical structure, physical properties and shapes [25].

It is well to mentioned that metal oxide NPs such as titanium dioxide NPs which used in sunscreen. When these products are gradually washed away by swimmers, nanoparticles accumulate in the water supply, which may affect aquatic food chains and can perhaps be led to unwanted toxic effects. It has been shown that titanium dioxide molecules cause damage to nervous cells in fish [10].

Viability assays assess the overall dosedependent toxicity of nanoparticles on cultured cells, looking for cell survival and proliferation after nanoparticle exposure. Toxicity of AuNPs was depended on their concentration, sizes and protecting ligands. For example, negative and positive AuNPs showed significant toxicity at concentrations of $0.5 \mathrm{nM}$ and $5 \mathrm{nM}$ of AuNP. The cationic AuNPs ( $2 \mathrm{~nm}$ in diameter) are toxic. However, the same NPs with a negatively charged surface was not toxic at the same concentration and in the same cell line [96]. AuNPs functionalised by alkylthiolate and tri (methyl) ammonium-ended ligands are more toxic than AuNPs functionalised by carboxylates ligands [97].

Other studies have been demonstrated that AuNPs less than $2 \mathrm{~nm}$ in diameter shown evidence of toxicity that does not occur at larger sizes [98]. ZnO NPs shown higher toxic effects on bacterial cells than other metal oxide NPs such as $\mathrm{TiO}_{2}$, due to their ionshedding ability.

Interestingly, ZnO-NPs have been reported in many studies as non-toxic to human cells. This aspect required its use as antibacterial agents, harmful to microorganisms and holding good biological compatibility of human cells [76]. High levels of zinc ions are the main contributors to ZnO NPs cellular toxicity, causing oxidative stress and inflammation. Different researches have shown that various shape of ZnO NPs such as rods and spheres are likely to neurotoxicity cause as result of its ability to reach the brain $[99,100]$.

\section{Conclusion}

Nanoparticles can be functionalized with a wide range of structural units using a simple chemical process under the moderate 
conditions. The stability of metal nanoparticles and metal oxide NPs by some kinds of ligands such as thiol and amines allows further manipulation of the usual compounds. Nanoparticles are expected to produce different chemical and optical properties which are used for potential uses as alternative biorecognition, drug delivery systems and catalyst. Thiols, phosphines, and amines have used as excellent functionalized compound for formation and stabilisation of the nanoparticles. Combination of the metal/metal oxide nanoparticle with these ligands would provide a variety of nanocomposites with controlled size. Also these ligands are the excellent candidates for this purpose due to their easy preparation. NPs can also act as a smart weapon against multidrug resistant microorganisms and an alternative approach to antibiotics. However, knowledge about the toxicity and health effect of NPs are essential for utilizing these nanoparticles in bio-applications.

It is expected that, the continued cooperation between chemist and nanotechnology which is desirable for producing the antibodies based on nanoparticles, will lead to the next medical revolution in the future.

\section{Acknowledgement}

The authors are thankful to Dr. Masoud Alragigthe (president of Sebha University) and Dr. Mohamed Erhayem (Vice President of Sebha University, Libya) for their support and kind encouragement.

\section{Disclosure statement}

No potential conflict of interest was reported by the author.

\section{References}

[1] K. Naka, Y. Chujo. Nanohybridization of organic-inorganic materials. Springer,
Berlin, Heidelberg, 2009, pp. 3-40.

[2] S.O. Obare, R.E. Hollowell, C.J. Murphy. Langmuir, 2002, 18, 10407-10410.

[3] Y. Kim, R.C. Johnson, J.T. Hupp, Nano Lett., 2001, 1, 165-167.

[4] S. Watanabe, M. Sonobe, M. Arai, Y. Tazume, T. Matsuo, T. Nakamura, K. Yoshida, Chem. Commun., 2002, 2002, 2866-2867.

[5] C.A. Mirkin, R.L. Letsinger, R.C. Mucic, J.J. Storhoff, Nature, 1996, 382, 607-609.

[6] S.Y. Lin, S.W. Liu, C.M. Lin, C.H. Chen, Anal. chem., 2002, 74, 330-335.

[7] G. Chumanov, K. Sokolov, B.W. Gregory, T.M. Cotton, J. Phys. Chem., 1995, 99, 9466-9471.

[8] S. Nie, S.R. Emory, Science., 1997, 275, 1102-1106.

[9] J. Shan, H. Tenhu, Chem. Commun., 2007, 2007, 4580-4598.

[10] L.L. Wang, C. Hu, L.Q. Shao, Int. J. nanomed., 2017, 12, 1227-1249.

[11] F. Ghandehari, M. Fani, M. Rezaee, J. Med. Chem. Sci., 2018, 1, 28-30.

[12] P. Schlexer, A.B. Andersen, B. Sebok, I. Chorkendorff, J. Schiøtz, T.W. Hansen, Particle Particle System. Characterizat., 2019, 36, 1800480.

[13] G. Thirumurugan, M. Dhanaraju, Antimicrobial Agents., 2012.

[14] F. Mohammadi, M. Yousefi, R. Ghahremanzadeh, Adv. J. Chem. Section A (Theoret., Eng. Appl. Chem.)., 2019, 2, 266-385.

[15] A. Abeer Mohamad, Int. J. Pharma. Bio. Sci., 2015, 6, 1357-1364.

[16] I. Sheikhshoaie, M. Sheikhshoaei, S. Ramezanpour, Chem. Method., 2018, 2, 83-180.

[17] A. Dehno Khalaji, Chem. Method., 2019, 3, 519-683.

[18] I. Amar, A. Sharif, M. Ali, S. Alshareef, F. Altohami, M. Abdulqadir, M. Ahwidi, Chem. Method., in Press.

[19] D. Mandal, M.E. Bolander, D. Mukhopadhyay, G. Sarkar, P. Mukherjee, 
Appl. Microbio. biotechnol., 2006, 69, 485492.

[20] Z. Duan, R. Sun, R. Liu, C. Zhu, Ene. Fuel., 2007, 21, 2056-2065.

[21] G. Zhao, S.E. Stevens, Biometals., 1998, 11, 27-32.

[22] C.Y. Lee, Y.T. Haung, W.F. Su, C.F. Lin, Appl. phys. lett., 2006, 89, 231116.

[23] Y.N. Slavin, J. Asnis, U.O. Häfeli, H. Bach, J. Nanobiotechnol., 2017, 15, 65.

[24] G.M. Patel, G.C. Patel, R.B. Patel, J.K. Patel, M. Patel, J. Drug Target., 2006, 14, 63-67.

[25] M. Fischman, V. Murashov, J. Borak, J. Seward. J. Occupant. Environ. Med., 2019, 61, e95-e98.

[26] R. Cao, R. Villalonga, A. Fragoso, IEE Proceed. Nanobiotechnol., 2005, 152, 159-164.

[27] D. Quintanar-Guerrero, E. Allémann, H. Fessi, E. Doelker, Drug develop. Indust. Pharm., 1998, 24, 1113-1128.

[28] L.G. Fernández, F. Puntes, B. Boix. PhD Thesis. Universitat Autònoma de Barcelona., 2013.

[29] J.A. Dahl, B.L.S. Maddux, J.E. Hutchison, Chem. Rev., 2007, 107, 2228-2269.

[30] M.C. Daniel, D. Astruc, Chem. Rev., 2004, 104, 293-346.

[31] H. Hirai, Y. Nakao, N. Toshima, J. Macromolecul. Sci. Chem., 1979, 13, 727750.

[32] H. Hirai, Y. Nakao, N. Toshima, J. Macromolecul. Sci. Chem., 1978, 12, 1117-1141.

[33] Y. Ju-Nam, W. Abdussalam-Mohammed, J. J. Ojeda, Faraday Discuss., 2016, 186, 7793.

[34] L. Jia, T. He, Z. Li, X.M. Li, Chin. J. Catal., 2010, 31, 1307-1315.

[35] J.M. Devi, J. Molecul. Grap. Model., 2017, 74, 359-365.

[36] J.W. Park, J.S. Shumaker-Parry, ACS Nano., 2015, 9, 1665-1682.

[37] G. Schmid, V. Maihack, F. Lantermann, S. Peschel, J. Chem. Soc., Dalton Trans., 1996, 1996, 589-595.
[38] P. Shanmugam, K. Rajakumar, R. Boddula, R.C. Ngullie, W. Wei, J. Xie, E. Murugan, Mater. Sci. Energy Technol., 2019, 2, 532542.

[39] G. Schmid, M. Harms, J.O. Malm, J.O. Bovin, J. Van Ruitenbeck, H.W. Zandbergen, W.T. Fu. J. Am. Chem. Soc., 1993, 115, 20462048.

[40] F.L. Wimmer, S. Wimmer, Inorg. Chim. Acta., 1988, 149, 1-3.

[41] G.H. Woehrle, L.O. Brown, J.E. Hutchison, J. Am. Chem. Soc., 2005, 127, 2172-2183.

[42] W.W. Weare, S.M. Reed, M.G. Warner, J. E. Hutchison, J. Am. Chem. Soc., 2000, 122, 12890-12891.

[43] D. Mahl, J. Diendorf, S. Ristig, C. Greulich, Z.A. Li, M. Farle, M. Köller, M. Epple, J. Nanopart. Res., 2012, 14, 1153.

[44] R.M. Lahtinen, S.F. Mertens, E. East, C.J. Kiely, D.J. Schiffrin, Langmuir., 2004, 20 , 3289-3296.

[45] Z. Khan, T. Singh, J.I. Hussain, A.A. Hashmi, Colloids and Surfaces B: Biointerfaces., 2013, 104, 11-17.

[46] X. Ji, X. Song, J. Li, Y. Bai, W. Yang, X. Peng, J. Am. Chem. Soc., 2007, 129, 1393913948.

[47] I. Ojea-Jiménez, F.M. Romero, N.G. Bastús, V. Puntes, J. Phys. Chem. C., 2010, 114, 1800-1804.

[48] A. Leifert, Y. Pan-Bartnek, U. Simon, W. Jahnen-Dechent, Nanoscale., 2013, 5, 6224-6242.

[49] M.A. Neouze, U. Schubert, Monat. ChemieChem. Mon., 2008, 139, 183-195.

[50] M.J. Rak, N K. Saadé, T. Friščić, A. Moores, Green Chem., 2014, 16, 86-89.

[51] H. Noorizadeh, A. Farmany, Adv. J. Chem. Section A., 2019, 2, 128-135.

[52] J. Cure, Y. Coppel, T. Dammak, P.F. Fazzini, A. Mlayah, B. Chaudret, P. Fau, Langmuir., 2015, 31, 1362-1367.

[53] E.D. Cavassin, L.F.P. de Figueiredo, J.P. Otoch, M.M. Seckler, R.A. de Oliveira, F.F. Franco, V.S. Marangoni, V. Zucolotto, A.S.S. Levin, S.F. Costa, J. nanobiotechnol., 
2015, 13, 64 .

[54] L.S. Dorobantu, C. Fallone, A.J. Noble, J. Veinot, G. Ma, G.G. Goss, R.E. Burrell, J. Nanopart. Res., 2015, 17, 172.

[55] E.S. Aazam, Z. Zaheer, Bioprocess Biosystem. Eng., 2016, 39, 575-584.

[56] X. Huang, X. Bao, Y. Liu, Z. Wang, Q. Hu, Sci. Rep., 2017, 7, 1860.

[57] M.M. Oliveira, D. Ugarte, D. Zanchet, A.J. Zarbin, J. Coll. Interface Sci., 2005, 292, 429-435.

[58] S. Sabella, A. Galeone, G. Vecchio, R. Cingolani, P.P. Pompa, J. Nanosci. Lett., 2011, 1, 145-165.

[59] J. Bennington-Castro, MRS Bull., 2016, 41, 178-179.

[60] S. Barua, S. Mitragotri, Nano Today., 2014, 9, 223-243.

[61] N. Padmavathy, R. Vijayaraghavan, Sci. Technol. Adv. Mater., 2008, 9, 035004.

[62] A.M. El Badawy, R.G. Silva, B. Morris, K.G. Scheckel, M.T. Suidan, T.M. Tolaymat, Environ. Sci. Technol., 2010, 45, 283-287.

[63] I.P. Mukha, A. Eremenko, N. Smirnova, A. Mikhienkova, G. Korchak, V. Gorchev, A.Y. Chunikhin, Appl. Biochem. Microbiol., 2013, 49, 199-206.

[64] L. Wang, H. He, Y. Yu, L. Sun, S. Liu, C. Zhang, L. He, J. Inorg. Biochem., 2014, 135, 45-53.

[65] V. Railean-Plugaru, P. Pomastowski, K. Rafinska, M. Wypij, W. Kupczyk, H. Dahm, M. Jackowski, B. Buszewski, Electrophoresis., 2016, 37, 752-761.

[66] H. Mu, J. Tang, Q. Liu, C. Sun, T. Wang, J. Duan, Sci. Rep., 2016, 6, 18877.

[67] A. Gupta, R.F. Landis, V.M. Rotello, F1000 Res., 2016, 5, 1-10.

[68] W. Zhang, Y. Li, J. Niu, Y. Chen. Langmuir., 2013, 29, 4647-4651.

[69] J. Gopal, M. Manikandan, N. Hasan, C.H. Lee, H.F. Wu, J. Mass Spectromet., 2013, 48, 119-127.

[70] A.A. Mohammed, Int. J. Curr. Microbiol. App. Sci., 2015, 4, 522-528.

[71] I. Sondi, B. Salopek-Sondi, J. Coll. Interface
Sci., 2004, 275, 177-182.

[72] L. Liu, J. Yang, J. Xie, Z. Luo, J. Jiang, Y.Y. Yang, S. Liu, Nanoscale., 2013, 5, 38343840.

[73] A. Grigor'eva, I. Saranina, N. Tikunova, A. Safonov, N. Timoshenko, A. Rebrov, E. Ryabchikova, Biometals., 2013, 26, 479488.

[74] C. Santos, A. Albuquerque, F. Sampaio, D. Keyson. Microbial pathogens and strategies for combating them: science, technology and education., 2013, 4, 143154.

[75] M.M. Mohamed, S.A. Fouad, H.A. Elshoky, G.M. Mohammed, T.A. Salaheldin, Int. J. Veter. Sci. Med., 2017, 5, 23-29.

[76] A. Sirelkhatim, S. Mahmud, A. Seeni, N.H.M. Kaus, L.C. Ann, S.K.M. Bakhori, H. Hasan, D. Mohamad, Nano-Micro Lett., 2015, 7, 219-242.

[77] B.V. Kumar, H.S.B. Naik, D. Girija, B.V. Kumar, J. Chem. Sci., 2011, 123 (5), 615621.

[78] (a) A. Nakhaei, A. Davoodnia, H. Nakhaei, J. Chem. Rev., 2019, 1, 139-153; (b) A. Nikam, T. Pagar, S. Ghotekar, K. Pagar, S. Pansambal, J. Chem. Rev., 2019, 1, 154163.

[79] K. Senthil, Y. Tak, M. Seol, K. Yong, Nanoscale Res. Lett., 2009, 4, 1329.

[80] S. Prashant, K. Kamlesh, C. Ramesh. J. Nanomed. Nanotechnol.., 2017.

[81] M. Nasrollahzadeh, S. Mahmoudi-Gom Yek, N. Motahharifar, M. Ghafori Gorab, Chem. Rec., 2019, 1-45.

[82] V.V. Torbina, A.A. Vodyankin, S. Ten, G.V. Mamontov, M.A. Salaev, V.I. Sobolev, O.V. Vodyankina, Catalysts, 2018, 8, 447.

[83] A. Murugadoss, A. Chattopadhyay, Nanotechnol., 2007, 19, 015603.

[84] S.V. Otari, R.M. Patil, N.H. Nadaf, S.J. Ghosh, S.H. Pawar, Environ. Sci. Pollut. Res., 2014, 21, 1503-1513.

[85] C. Deraedt, L. Salmon, S. Gatard, R. Ciganda, R. Hernandez, J. Ruiz, D. Astruc, Chem. Commun.., 2014, 50, 14194- 
14196.

[86] X. Liu, J. Ruiz, D. Astruc, J. Inorg. Org. Polym. Mater., 2018, 28, 399-409.

[87] S. Liu, Z. Zhang, Y. Wang, F. Wang, M.Y. Han, Talanta., 2005, 67, 456-461.

[88] N. Berahim, W. Basirun, B. Leo, M. Johan, Catalysts, 2018, 8, 412.

[89] X. Fang, Y. Wang, Z. Wang, Z. Jiang, M. Dong, Energies, 2019, 12, 190.

[90] N.K.R. Bogireddy, H.A.K. Kumar, B.K. Mandal, J. Environ. Chem. Eng., 2016, 4, 56-64.

[91] Y. Yuan, N. Yan, P.J. Dyson, Inorg. Chem.., 2011, 50, 11069-11074.

[92] B.L.V. Prasad, S.I. Stoeva, C.M. Sorensen, V. Zaikovski, K.J. Klabunde, J. Am. Chem. Soc., 2003, 125, 10488-10489.

[93] S. Wang, Q. Zhao, H. Wei, J.Q. Wang, M. Cho, H. S. Cho, O. Terasaki, and Y. Wan, J. Am. Chem. Soc.,2013, 135, 11849-11860.
[94] S. Alizadeh, T. Madrakian, M. Bahram, Adv. J. Chem. Section A., 2019, 2, 57-72.

[95] A. Sharma, S.V. Madhunapantula, G.P. Robertson, Expert Opin. Drug Metabol. Toxicol., 2012, 8, 47-69.

[96] C.M. Goodman, C.D. McCusker, T. Yilmaz, V.M. Rotello, Bioconjugate Chem., 2004, 15, 897-900.

[97] A.M. Alkilany, C.J. Murphy, J. Nanoparticle Res., 2010, 12, 2313-2333.

[98] C.L. Villiers, H. Freitas, R. Couderc, M.B. Villiers, P.N. Marche, Journal of Nanoparticle Research., 2010, 12, 55-60.

[99] J. Liu, Y. Kang, S. Yin, B. Song, L. Wei, L. Chen, L. Shao, Int. J. Nanomed., 2017, 12, 8085-8099.

[100] S. Taghavi Fardood, A. Ramazani, F. Moradnia, Z. Afshari, S. Ganjkhanlu, F. Yekke Zare, Chem.Methodol., 2019, 3, 696-706.

How to cite this manuscript: Wanisa Abdussalam-Mohammed, Comparison of Chemical and Biological Properties of Metal Nanoparticles (Au, Ag) with Metal Oxide Nanoparticles (ZnO-NPs) and their Applications, Adv. I. Chem. A, 2020, 3(2), 192-210. 